\title{
Successful Rechallenge with Osimertinib after Very Acute Onset of Drug-Induced Pneumonitis
}

\author{
Turab Mohammed $^{a}$ Shaunak Mangeshkar ${ }^{b} \quad$ Joerg Rathmann ${ }^{c}$ \\ aDepartment of Medicine, University of Connecticut School of Medicine, Farmington, CT, \\ USA; bepartment of Medicine, Seth G.S. Medical College and K.E.M. Hospital, Mumbai, \\ India; 'Division of Hematology and Oncology, Hartford Healthcare Care Institute, Hartford \\ Hospital, Hartford, CT, USA
}

\section{Keywords}

Osimertinib · Drug-induced pneumonitis · Drug-induced ILD · Transient asymptomatic pulmonary opacities $\cdot$ Non-small cell lung cancer

\begin{abstract}
Drug-induced interstitial lung disease (DI-ILD) is a rare, yet life-threatening complication associated with tyrosine-kinase inhibitor (TKI) therapy. Third-generation epidermal growth factor receptor-TKI, osimertinib use can be associated with a benign radiological finding called transient asymptomatic pulmonary opacities that can be confused with an infectious pulmonary process resulting in overtreatment with antibiotics or premature treatment withdrawal or severe DI-ILD. In this case, our patient with newly diagnosed metastatic non-small cell lung cancer on treatment with osimertinib developed very early onset severe DI-ILD (grade-IV) with a unique pattern of pulmonary involvement and was treated with high-dose corticosteroids with a response. She was later successfully rechallenged with osimertinib and responded well to the treatment. Our case highlights the importance of being cognizant of the possibility that DI-ILD can rarely occur within a week of treatment initiation with osimertinib and safe reintroduction of the drug is possible in select patients following complete resolution of pulmonary radiographic findings and clinical symptoms even with high-grade adverse events.

(c) 2021 The Author(s).

Published by S. Karger AG, Basel
\end{abstract}

\section{Introduction}

Targeted therapy against epidermal growth factor receptor (EGFR) driver mutations, the most frequently documented gene mutation in non-small cell lung cancer (NSCLC), is now the frontline standard-of-care for patients with advanced-stage disease harboring this specific

\section{Karger!}


mutation [1]. Osimertinib, a third-generation irreversible EGFR tyrosine-kinase inhibitor (TKI) has been used for treatment-resistant NSCLC and was recently approved for patients with advanced NSCLC for first-line use [2]. Drug-induced pneumonitis is a rare, yet life-threatening complication associated with EGFR kinase inhibition therapies including osimertinib [3]. Its use can be associated with a unique and benign radiological finding called "Transient Asymptomatic Pulmonary Opacities (TAPO)" that can be confused with an infectious pulmonary process or drug-induced interstitial lung disease (DI-ILD) and may result in overtreatment with antibiotics or premature treatment withdrawal [4]. Here, we present the case of a patient with newly diagnosed metastatic NSCLC started on treatment with osimertinib who developed very early onset severe drug-induced pneumonitis with a unique pattern of pulmonary involvement and was treated with corticosteroids. After resolution of her pulmonary findings, the patient was successfully rechallenged with osimertinib and continued to have an antitumor response.

\section{Case Description}

A 71-year-old non-Hispanic white female, never-smoker was incidentally diagnosed with advanced-stage NSCLC after presenting with acute right-sided shoulder pain secondary to a pathological fracture of the humerus from a metastatic lytic lesion. Diagnostic computed tomography (CT) images demonstrated right lower lobe lung mass, with widespread lymphadenopathy and multiple lytic lesions (Fig. 1a). Diagnostic biopsy of the humeral lesion revealed a lung adenocarcinoma and the immune-histochemistry was positive for CK-7, TTF-1, Napsin A, and PAX-8. Molecular testing revealed 0\% PDL-1 expression, mutation testing negative for ALK and ROS1 but positive for EGFR exon 19 deletion (E19del) driver mutation. She was diagnosed with Stage IV lung cancer (cT3, cN2, and pM1) and started on osimertinib $80 \mathrm{mg}$ tablet once a day as a first-line agent based on molecular tumor profiling.

After 1 week on the therapy, she developed rapidly progressive shortness of breath and was hospitalized for acute hypoxic respiratory failure. On physical examination, her vital signs were remarkable for tachypnea, tachycardia, and decreased breath sounds bilaterally. Differential diagnoses included community-acquired pneumonia, COVID-19 pneumonia, cardiogenic pulmonary edema, acute pulmonary embolism, progressive disease, lymphangitic carcinomatosis, and drug-induced pneumonitis as a diagnosis of exclusion (Table 1). Laboratory workup revealed mild leukocytosis (WBC-12,500/ $\mu \mathrm{L}$ ) with left shift, lactate of 2.5 $\mathrm{mmol} / \mathrm{L}$ (normal - <2), C-reactive protein (CRP) - $11.7 \mathrm{mg} / \mathrm{dL}$ (normal $-<0.5$ ), procalcito-
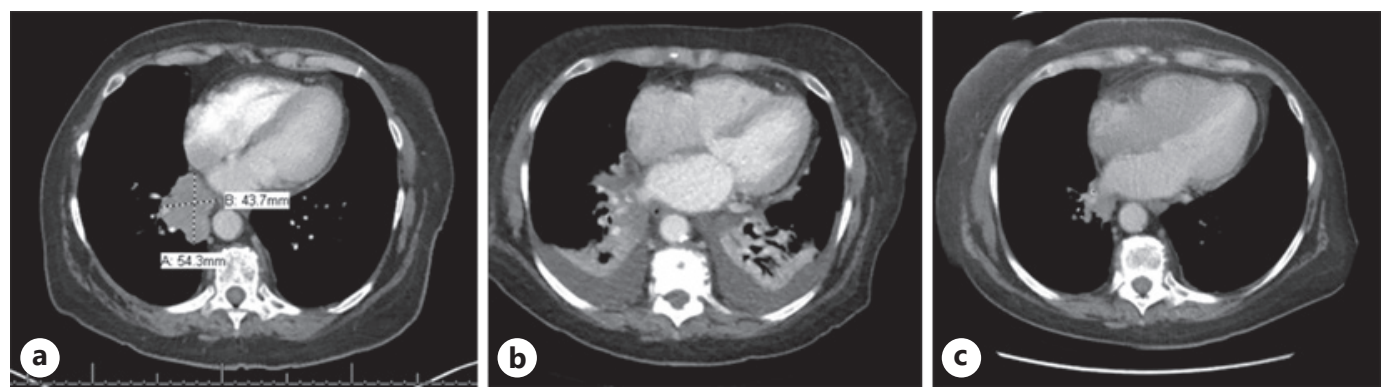

Fig. 1. a-c CT scan of the chest showing the right lower lobe lung mass at the time of cancer diagnosis in a. Repeat CT scan demonstrating bilateral lower lobe consolidation with air bronchograms and new-onset bilateral pleural effusion in b. CT scan after 6 weeks of corticosteroid therapy showing complete resolution of the lung findings in c. CT, computed tomography. 
Table 1. Differential diagnosis for acute pulmonary symptoms in patients with lung cancer

Primary tumor progression

Metastatic disease (lymphangitic carcinomatosis, malignant pleural effusion)

Acute pulmonary embolism

Infection

Cardiogenic pulmonary edema

Pulmonary hemorrhage

Acute respiratory distress syndrome

Transfusion-associated circulatory overload (TACO)

Transfusion-related acute lung injury (TRALI)

Drug-induced pneumonitis

Fig. 2. Chest X-Ray demonstrating bibasilar patchy airspace opacities predominantly involving the right lower lobe.

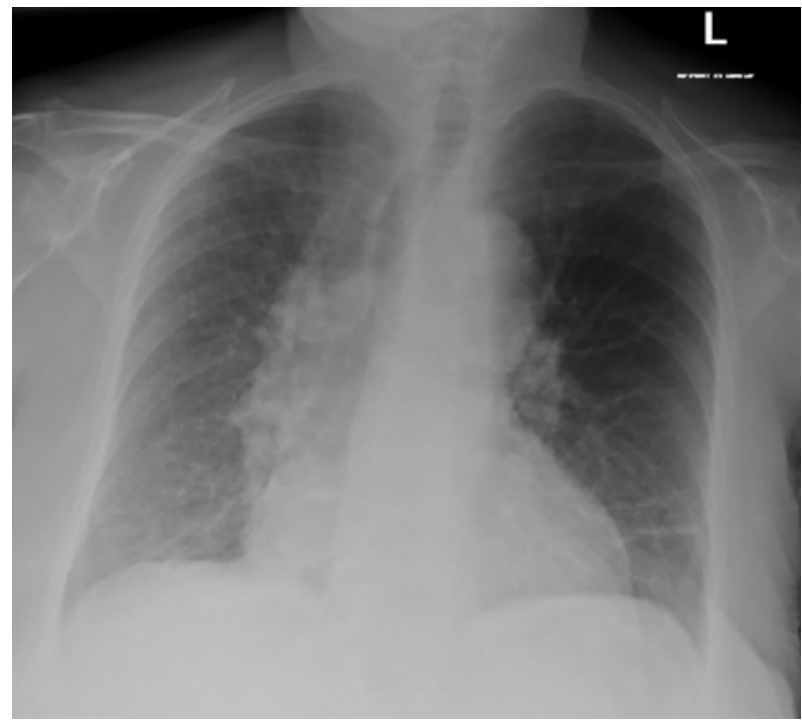

nin - $0.2 \mathrm{ng} / \mathrm{mL}$ (normal - <0.09), new-onset atrial fibrillation on EKG, negative COVID-19 PCR test and normal age-adjusted D-Dimer levels. Chest X-ray demonstrated bibasilar patchy airspace opacities predominantly involving the right lower lobe (Fig. 2) and echocardiogram revealed normal ejection fraction. She was started on empiric broad-spectrum antibiotic therapy with vancomycin and cefepime for suspected pneumonia with sepsis and the osimertinib was withheld temporarily. Over the next few days, her respiratory failure continued to worsen despite the antibiotics. Chest CT scan now demonstrated bilateral dense lower lobe consolidation with air bronchograms and new-onset bilateral pleural effusion but a decrease in the size of dominant lung mass (Fig. 1b). She was started on high-dose corticosteroids for suspected drug-induced pneumonitis and her symptoms improved significantly within $24 \mathrm{~h}$ of starting steroid therapy. Subsequently, all antibiotics were discontinued, and she was discharged home on corticosteroid taper and with osimertinib drug being held.

Repeat CT scan at 6 weeks from discharge revealed near-total resolution of the infiltrates and pleural effusions (Fig. 1c). Given the impressive response of patient's lung cancer to osimertinib, complete resolution of pulmonary symptoms without any further complications, and literature review supporting the successful reintroduction of the drug among some patients despite a history of pulmonary side effects, osimertinib was cautiously restarted at 
half the standard dose and gradually escalated to the full dose of $80 \mathrm{mg}$ daily. The patient tolerated drug reintroduction well, has remained asymptomatic, and continues to have antitumor benefit.

\section{Discussion/Conclusion}

Osimertinib is the treatment of choice for patients with NSCLC with acquired T790M resistance, and first-line treatment in patients with exon 21 L858R and exon 19 mutations [5]. It has also been linked to a wide range of pulmonary complications ranging from asymptomatic opacities/infiltrates on imaging called TAPO [4] to catastrophic diffuse alveolar hemorrhage [6]. DI-ILD has been associated with EGFR-TKI therapies, including osimertinib in $1-3 \%$ of patients with reported mortality in up to $22 \%$ of the patients [7]. The proposed mechanisms for osimertinib-induced lung injury include immune-mediated inflammation and cytotoxic injury by impairing antiapoptotic mechanisms [8]. Detecting TAPO in patients on osimertinib therapy may signify either a benign process or the initial stages of evolving pneumonitis [4].

Our patient demonstrated very early onset symptom manifestation and her severe grade 3 ILD with unique striking pulmonary findings responded well to high-dose corticosteroids. We could find only one case in the literature where osimertinib-related pulmonary complications occurred within 1 week of treatment initiation [9]. A unique finding in our patient was the presence of pleural effusion, a finding not commonly reported with EGFR TKI-related ILD. To our knowledge, we could not find any study or individual report referencing pleural effusion as a finding in the ILD spectrum-related with this class of drugs. Whether lung opacities and effusion were initially the TAPO that progressed to ILD is not known. Studies suggest that TAPO could be the preceding finding before the osimertinib-induced ILD in some patients $[4,10]$. Lee et al. [10] have classified asymptomatic pulmonary opacities into distinct radiological subtypes based on analysis of CT scans of patients with lung cancer treated with osimertinib. These include cryptogenic organizing pneumonia, simple eosinophilic pneumonia, and the nodular subtype, and others (Table 2).

The management of pulmonary complications in patients receiving osimertinib can be challenging in terms of the decision to rechallenge, switch to alternative TKI or choose a waitand-watch strategy, given the possibility of transformation of TAPO into symptomatic or even fatal pulmonary complications. Among patients with symptomatic disease, the subsequent progression of TAPO on follow-up and progressive diffuse alveolar damage pattern on imaging, immediately withholding osimertinib and, providing supportive therapy along with a course of corticosteroids has been found to be effective $[10,11]$.

The current evidence-based practice is to discontinue oral targeted therapy after an episode of severe drug-induced pneumonitis. A "Stop-and-Go" technique which comprises drug discontinuation followed by rechallenge has been employed as a reasonable option of

Table 2. Various patterns of pulmonary opacities described in patients while on osimertinib therapy
Ground glass opacities
Organizing pneumonia
SEP

Subpleural nodule

Peribronchial nodule

Nonspecific interstitial pneumonia

SEP, simple eosinophilic pneumonia. 
managing non-metastatic pulmonary opacities occurring during treatment with osimertinib with a documented antitumor response. Kobayashi et al. [11] suggested stopping treatment upon the development of early pulmonary opacities, such as GGOs as they may denote the late onset of life-threatening ILD. Kobayashi et al. [11] recommend 6 weeks of "drug holiday" as it is the median time for resolution of TAPO before osimertinib rechallenge can be considered. While ensuring very close follow-up in the clinic, we successfully rechallenged our patient with osimertinib after the complete resolution of clinical and radiographic lung findings after 6 weeks of corticosteroid therapy. In conclusion, our case highlights the possibility that DI-ILD can rarely occur within days of treatment initiation with osimertinib and safe reintroduction of the drug is possible in select patients following complete resolution of pulmonary radiographic findings and clinical symptoms even with high-grade adverse events.

\section{Statement of Ethics}

The subject of this case report gave her written, informed consent to publish the case, including the publication of images. No institutional review board or other approvals were required since it is de-identified.

\section{Conflict of Interest Statement}

The authors report no conflicts of interest.

\section{Funding Source}

The authors did not receive any funding.

\section{Author Contributions}

Turab Mohammed: provided care to the patient, collected patient information, reviewed literature, and wrote the manuscript. Shaunak Mangeshkar: collected patient information, reviewed literature, and wrote the manuscript. Joerg Rathmann: medical oncologist on the team, supervised care of the patient, and provided critical inputs for the final manuscript.

\section{References}

1 Soria JC, Ohe Y, Vansteenkiste J, Reungwetwattana T, Chewaskulyong B, Lee KH, et al. Osimertinib in untreated EGFR-mutated advanced non-small-cell lung cancer. N Engl J Med. 2018;378(2):113-25.

2 Research C for DE. FDA approves osimertinib as adjuvant therapy for non-small cell lung cancer with EGFR mutations. FDA. 2020. Available from: https://www.fda.gov/drugs/drug-approvals-and-databases/fdaapproves-osimertinib-adjuvant-therapy-non-small-cell-lung-cancer-egfr-mutations Accessed 2021 Jan 28.

3 Häntschel M, Niebling J, Häring A, Häring MF, Groß T, Horger M, et al. Life-threatening pneumonitis after firstline treatment with osimertinib for primary T790M mutated non-small cell lung cancer. Thorac Cancer. 2020; 11(7):2044-7.

4 Noonan SA, Sachs PB, Camidge DR. Transient asymptomatic pulmonary opacities occurring during osimertinib treatment. J Thorac Oncol. 2016;11(12):2253-8.

5 Wu SG, Shih JY. Management of acquired resistance to EGFR TKI-targeted therapy in advanced non-small cell lung cancer. Mol Cancer. 2018;17(1):38.

6 Forte MJ, Sangani RG. Emerging risk profile of lung cancer therapy: diffuse alveolar hemorrhage from osimertinib. Case Rep Oncol Med. 2019;2019:6185943. 
7 Ohmori T, Yamaoka T, Ando K, Kusumoto S, Kishino Y, Manabe R, et al. Molecular and clinical features of EGFRTKI-associated lung injury. Int J Mol Sci. 2021;22(2):792.

8 Lu H, Dowell J. Osimertinib in pulmonary manifestations: two case reports and review of the literature. In Vivo. 2020;34(1):315-9.

9 Ahn JH. Successful osimertinib retreatment after extremely early onset severe pneumonitis in first-line treatment of lung adenocarcinoma. Thorac Cancer. 2020;11(9):2713-6.

10 Lee H, Lee HY, Sun JM, Lee SH, Kim Y, Park SE, et al. Transient asymptomatic pulmonary opacities during osimertinib treatment and its clinical implication. J Thorac Oncol. 2018;13(8):1106-12.

11 Kobayashi K, Naoki K, Kuroda A, Yasuda H, Kawada I, Soejima K, et al. EGFR-mutant non-small cell lung cancer accompanied by transient asymptomatic pulmonary opacities successfully treated with "stop-and-go" osimertinib. Intern Med. 2018;57(7):1007-10. 